\title{
La crisis, la geografía económica y Julie Graham: Alternativas de desarrollo local a partir de la crítica feminista ${ }^{1}$
}

\author{
Antònia CASELLAS \\ Departament de Geografia Universitat Autònoma de Barcelona \\ antonia.casellas@uab.cat
}

Recibido: 9 de Noviembre de 2010

Enviado a evaluar: 2 de Marzo de 2011

Aceptado: 28 de Junio de 2011

\section{RESUMEN}

El presente artículo reflexiona sobre interpretaciones académicas consolidadas y estrategias dominantes en desarrollo local, contraponiéndolas al potencial que ofrece el concepto de desarrollo local fundamentado en principios éticos y políticos. El artículo se organiza a través de un doble objetivo. Por un lado se desea rendir tributo al trabajo de Julie Graham, profesora de geografía económica de la Universidad de Massachusetts en Amherts que murió repentinamente en el 2010. Por otro lado, tomando como pretexto el legado de Graham, se amplia y desarrolla su línea de pensamiento con las aportaciones de enfoques feministas, posestructuralistas y neo-marxistas, con el fin de avanzar en la exploración de claves interpretativas que permitan crear una nueva narrativa sobre desarrollo. Se concluye que las alternativas a la presente crisis pueden encontrarse en las potencialidades que ofrecen las economías locales si éstas están enraizadas en el territorio y se fundamentan en cuestiones éticas y de justicia social y medioambiental.

Palabras clave: desarrollo, economía local, comunidad, globalización, geografía económica, Julie Graham.

The crisis, the economic geography and Julie Graham: local development alternatives from the feminist critique

\begin{abstract}
This article reflects on academic interpretations consolidated and well established local development strategies, weighed against the potential that offers the concept of local development based on ethical and political principles. The article is organized through a dual purpose. On one hand it wishes to pay tribute to the work of Julie Graham, professor of economic geography at the University of Massachusetts at Amherst who passed away suddenly in 2010. On the other hand, on the pretext of Graham's legacy,
\end{abstract}

${ }^{1}$ La investigación que da lugar a la presente aportación se inscribe en el Subprograma Ramón y Cajal del Ministerio de Ciencia e Innovación con la referencia RYC-2008-02456; y en los Programas Generales de Investigación y Desarrollo del Ministerio de Ciencia e Innovación, referencias CSO200908271 y CSO2010-17178. 
it extends and develops its thinking with the contributions of feminist, poststructuralist and neo-Marxist approaches, to advance towards interpretative approaches for building a new development narrative. It concludes that alternatives to the present crisis can be found in the potential offered by local economies if these are rooted in the territory and based on ethical questions and social and environmental justice.

Key words: development, local economy, community, globalization, economic geography, Julie Graham

La crise, la géographie économique et Julie Graham: des alternatives au développement local à partir de la critique féministe

\section{RÉSUMÉ}

Cet article porte sur les interprétations académiques consolidées et les stratégies dominantes du développement local, confrontant celles-ci avec le potentiel du concept de développement local fondé sur des principes éthiques et politiques. L'article s'organise autour d'un double objectif. D'une part, il tient à rendre hommage au travail de Julie Graham, professeur de géographie économique à l'Université du Massachusetts à Amherst, décédée subitement en 2010. D'autre part, sous le prétexte d'éclairer l'héritage de Graham, l'article prolonge et développe la réflexion de cet auteur à travers certaines contributions féministes, post-estructuralistes et néo-marxistes pour ainsi faire progresser l'exploration de clés interprétatives dans le but de construire un nouveau récit du développement. Il conclut que les alternatives à la crise actuelle se situent dans le potentiel présent au niveau des économies locales, à condition que ces dernières soient enracinées dans le territoire et fondées sur l'idéal de justice et d'éthique sociale et environnementale.

Mots clef: développement, économie locale, communauté, mondialisation, géographie économique, Julie Graham

\section{INTRODUCCIÓN}

Una de las preguntas que podemos formularnos desde la geografía económica es qué posibilidad de respuesta se puede aportar, desde este ámbito académico, a la crisis que viven las economías avanzadas. Este artículo pretende ofrecer propuestas interpretativas a esta pregunta. Una pregunta que no nace de la voluntad de contribuir a la recuperación del modelo capitalista que ha entrado en crisis, sino de investigar y explorar posibles alternativas al fallido modelo, desde nuevas líneas de análisis y, en especial, a partir de contribuciones claves de Julie Graham, profesora de geografía económica del Departamento de Geociencias de la Universidad de Massachusetts en Amherts que murió repentinamente en abril del 2010.

En un periodo con una gran necesidad de reflexionar sobre modelos económicos de desarrollo local alternativos, los análisis e interpretaciones que Graham y su línea de pensamiento hacen de la economía y el espacio económico son extraordinariamente valiosos. En este sentido, el objetivo del presente artículo es doble. Por un lado se trata de rendir tributo al trabajo de Graham, y por otro lado se desea, tomando como pretexto su legado, ampliar y desarrollar su línea de pensamiento con las aportaciones de otros pensadores, para generar claves interpretativas que permitan buscar alternativas a los modelos de desarrollo local imperantes. 
El presente artículo se estructura por ello siguiendo la evolución académica de Graham, extendiendo y ampliando sus análisis con la aportación de otros pensadores. En los siguientes apartados se critica, en primer lugar, las limitaciones de los estudios del ámbito teórico marxista para generar alternativas viables al sistema capitalista que cuestionan. En especial se enfatiza la crítica que Graham hace al marxismo desde la perspectiva posestructuralista, en cuanto este enfoque permite a Graham identificar cómo el análisis marxista tradicional en geografía económica, y por extensión en otros ámbitos del saber, se ha caracterizado por ser una narrativa esencialista incapaz de abrirse a nuevos discursos y perspectivas analíticas más fructíferos. A continuación se expone las restricciones que Graham y su línea de pensamiento identifican en el otro modelo económico dominante. En este caso el que, desde el espectro ideológico opuesto al marxismo, ha operado como el marco teórico básico del sistema capitalista, el de los economistas clásicos. Estos han identificado durante décadas la teoría de la base económica como el modelo ideal de crecimiento económico. Por ello la siguiente sección analiza los orígenes y conceptos básicos de esta teoría con larga tradición y arraigo entre un amplio abanico de académicos, consultores y políticos. Se identifica las limitaciones de este enfoque y se explora el porqué y cómo esta teoría ha generado un determinado concepto del crecimiento local, menoscabando otros procesos económicos mejor vinculados al territorio.

La crítica feminista y las interpretaciones surgidas desde la teoría queer permiten identificar nuevas vías de análisis de la realidad económica. Para desarrollar este punto, la sección tercera hace un repaso a pensadores feministas y sus contribuciones. Por un lado, se ejemplifica el cuestionamiento que desde este enfoque se hace de variables y conceptos económicos clásicos; y por otro lado se ofrece nuevas líneas de apertura hacia análisis teóricos innovadores y herramientas metodológicas que permitan capturar procesos económicos que a menudo son ignorados, como por ejemplo, los referentes al trabajo de las mujeres. Desde la apertura que implica la mirada feminista y que, la siguiente sección expone el trabajo conjunto de Graham and Gibson, con especial atención a dos de sus publicaciones más emblemáticas, The End of Capitalism (as we knew it) y A Postcapitalist Politics, los cuales argumentan y exploran la existencia de espacios económicos alternativos a los identificados por la narrativa capitalista. El caso específico de Mondragón ilustra este análisis.

Finalmente la última sección apunta a posibles respuestas partiendo de la posibilidad de identificar aquellas actividades económicas que siendo viables y sostenibles para las economías locales pasan desapercibidas, cuando no, menoscabadas, en las interpretaciones economicistas tradicionales y en la narrativa marxista. Partiendo de la crítica al concepto de desarrollo sostenible que han desarrollado diferentes autores, el último capítulo resume las contribuciones de Julie Graham entorno a las potencialidades de las economías locales enraizadas en el territorio y motivadas por cuestiones éticas y de justicia social y medioambiental. 


\section{LA CRÍTICA POSESTRUCTURALISTA AL DISCUSO MARXISTA}

Desde la obtención de su Doctorado en Geografía Económica en Clark University en 1984, Julie Graham dedicó su carrera académica y su experiencia vital a repensar las dinámicas de la economía, entre ellas los conceptos que rigen las interpretaciones económicas. Desde suposicióninicial comoeconomista política de tradición anglosajona, Graham consideró que la economía no podía interpretarse separadamente de la política y de las relaciones de poder. El desarrollo de las ciudades y sus modelos productivos deberían, pues, entenderse considerando que las decisiones tomadas por individuos y organizaciones que poseen recursos son claves para determinar las características del modelo productivo, que la estructura y el crecimiento o decrecimiento de las ciudades están vinculados a los procesos de restructuración económica, y que el estado juega un papel fundamental como agente de transformación. Esta línea de pensamiento la situó en el marco de la geografía crítica o marxista.

Sin embargo, Graham estuvo también conectada desde el inicio al pensamiento feminista y posestructuralista, y desde este posicionamiento dirigió pronto una dura crítica al marxismo tradicional. La postura de Graham surge de su necesidad de buscar nuevas estrategias interpretativas al proceso de transformación económica de los territorios, con sus repercusiones espaciales y sociales. Dada la incapacidad del marxismo de proponer nuevas propuestas de pensamiento y acción, desde la crítica feminista y los postulados posestructuralistas, Graham, a menudo junto a Katherine Gibson, atacará las limitaciones del marxismo (Gibson-Graham et al, 2000; GibsonGraham, et al 2001). En este objetivo, mantiene una posición epistemológica similar a la de Foucault (1980) cuando éste critica la Modernidad y la Ilustración por ser nuevos modos de dominación que imponen su racionalidad; y a las teorías dialécticas como el marxismo, por ser teorías totalizadoras, reduccionistas y coercitivas. En esta línea de análisis, a finales de los años ochenta Graham presiona a los geógrafos marxistas a abandonar su esencialismo y a interpretar el marxismo como tan solo "una forma" de interpretar el mundo.

En su emblemático artículo publicado en Antipode en 1988, Graham escribía:

The post-modern movement carries a serious critical message that Marxists should consider. The general critique that post-modernism offers to established modern discourses is the critique of essentialism. With respect to Marxism, this critique focuses on the Marxist illusion that class (or production, or accumulation) is the fundamental ground of reality rather than a social process constituted as a focal theoretical category within one of many competing discourses (Graham, 1988: 62).

El movimiento post-moderno conlleva un serio mensaje de crítica que los marxistas deberían tener en consideración. La crítica general que ofrece el post-modernismo a los discursos modernos establecidos es la crítica al esencialismo. Con respecto al marxismo, esta crítica se centra en la ilusión marxista que la clase social (o la producción, o la acumulación) es la base fundamental de la realidad, en lugar de un proceso social constituido como una categoría teorética central dentro de uno de los muchos discursos que compiten entre ellos (Graham, 1988: 62 -Traducción del autor). 
El esfuerzo por liberar a los pensadores marxistas, tan influyentes en la geografía anglosajona, de su esencialismo implica un esfuerzo por ampliar las categorías que se usan en el análisis de las realidades económicas. Implica también liberar el análisis en geografía económica de las limitaciones que supone interpretar procesos históricos complejos con un limitado número de categorías, y también, como Graham apunta, de esperar cambios políticos en lugar de hacer política. Graham enfatiza que los enfoques posmodernos no son necesariamente perspectivas conservadoras. En su opinión no se trata de negar la importancia de las relaciones de clase, sino de resistirse a subordinar toda la experiencia vital y social, a la lucha de clases y a las leyes de acumulación capitalista, tal como lo hace el análisis marxista.

$\mathrm{Su}$ argumento es extraordinariamente poderoso, ya que es una crítica desde dentro. Graham se puede identificar como economista política. Por ello su aportación es especialmente relevante. A través de su crítica al esencialismo marxista, Graham empieza a elaborar ya el argumento que más tarde desarrollara junto a Gibson, arguyendo que es precisamente el análisis marxista el que aporta la narrativa legitimadora al modelo capitalista. En la cuarta sección de este artículo retomamos este punto.

\section{LA GLOBALIZACIÓN, LA DESINDUSTRIALIZACIÓN Y LAS LIMITACIONES DE LA TEORIA DE LA BASE ECONÓMICA}

A Graham le preocupa especialmente el impacto que el proceso de globalización tiene en la economía y en la clase trabajadora. En los años ochenta, los EEUU sufren una transformación radical de su economía. Es una transformación muy rápida que afecta especialmente las ciudades industrializadas del norte, lo que popularmente se conoce como el rust belt, los Estados del Noreste donde tuvo lugar el proceso de industrialización del país. La globalización implica una transformación del modelo productivo que, como también sucederá en Europa un poco más tarde, se llevará a cabo inicialmente a través de la reubicación de plantas productivas en el exterior del país (offshoring), y la subcontratación de la producción en el exterior (offshore outsourcing), con la consecuente desindustrialización para el país de origen y la masiva pérdida de puestos de trabajo.

El sueño americano de clase media y trabajadora desaparece bajo un rápido deterioro de las condiciones laborales. Se produce un declive muy significativo de puestos de trabajo, que se agudiza aún más en los años noventa, para acentuarse a consecuencia de la crisis del 2001. Desde el 2000 al 2003, más de 3 millones de trabajos en el sector manufacturero desaparecen en los EEUU, a la vez que empiezan a decrecer también muchos puestos de trabajo en el sector servicios (Arnesen, 2007). Un proceso que, como ya hemos apuntado anteriormente, también tendrá su réplica en Europa, con paralelismos muy similares. Estudios de casos concretos han documentado el fuerte impacto en las economías locales que implica la pérdida de empleo masivo en el sector industrial (Kirsch 1998; Teaford 1994). 
El desconcierto social que este proceso genera en los Estados Unidos lo ilustra Michael Moore en su película de 1989 Roger \& Me. En ella Moore analiza la crisis económica, urbana, y social que sufrió su ciudad natal de Flint, situada en el corazón industrial de Michigan. El cierre de la fábrica de General Motors (GM) en 1983 dejó a unas 30.000 personas sin trabajo. De forma autobiográfica el director recoge la conmoción social y humana que vivió su ciudad. Como hijo de un trabajador en una línea de montaje de GM, Moore afirma que su generación creció bajo la creencia de que si se trabaja con dedicación para una empresa, y ésta prospera, los trabajadores, sus familias y su comunidad iban necesariamente a beneficiarse. El argumento de la película de Moore es pedir explicaciones a Roger Smith, por aquel entonces presidente de GM de porqué la compañía cierra la planta de Flint para reubicar su producción en Méjico a principios de los años ochenta, considerando que en aquel periodo GM tenía un superávit millonario. A lo largo del film, y a pesar de sus esfuerzos, Michael Moore no conseguirá hablar con el presidente de GM. Pero, tal y como uno los representantes de GM argumentan en la película, la respuesta desde la empresa se encuentra en la necesidad que ésta tiene de aprovechar las oportunidades que ofrece la globalización de la economía.

El film de Moore también recoge como la clase política y económica de Flint intentará desesperadamente reposicionar económicamente la ciudad después del cierre de GM. Para ello, las políticas de promoción económica en Flint reproducirán una lógica ampliamente aceptada. Dado que el proceso de globalización es irreversible, lo que las ciudades deben hacer es insertarse en la economía mundial de manera que puedan conseguir nueva actividad económica con la que competir en un mundo globalizado. Frente de la desindustrialización, las ciudades intentarán posicionarse como centros culturales, creativos e innovadores a escala global (Florida, 2002; Moses 2001; Fulton and Shigley, 2001; Gottleib, 1995). Este es un argumento que de forma insaciable han defendido desde los años ochenta y desde diferentes perspectivas, tanto en los EEUU como en Europa, numerosos políticos locales y geógrafos económicos.

Retomando el análisis de Julie Graham, y tal como sus estudios señalan, el crecimiento económico desde la teoría de la base económica tiene fuertes limitaciones. Graham apunta que la creencia ampliamente aceptada entre académicos y agentes en desarrollo local y regional ha sido considerar que la única manera de evitar el colapso de las economías locales en un contexto de globalización de la economía es el desarrollo de productos y servicios para la demanda externa; en las últimas décadas, ligada a la economía del conocimiento y al ocio a través de la especialización en tecnología, turismo, enseñanza superior, etc. El argumento de la base económica o teoría de los multiplicadores se centra en considerar que cada puesto de trabajo que creamos en una industria de demanda externa produce empleo local en otros sectores a través del efecto multiplicador (Archer, 1976).

La teoría de la base económica fue utilizada inicialmente por la planificación y el análisis regional en la década de 1930. Fue adoptado en la década de los años 1950 por los economistas urbanos, aplicando la teoría de crecimiento macroeconómico al estudio de las zonas urbanas a través del modelo de desarrollo regional keynesiano (Goodwin, 1949; Metzler, 1950; Chipman, 1950; Isard, 1960). Los vínculos entre 
estas fuentes fueron discutidas en detalle por Charles Tiebout (1956a, 1956b). La teoría postula que el crecimiento y desarrollo de una zona geográfica está determinada en función de la demanda externa de bienes y servicios originada fuera de dicha zona. En este sentido, las industrias "exportan", y a la producción de bienes y servicios de la demanda externa se les denomina la "base económica" del área geográfica.

La idea central de la teoría es la suposición de que toda la actividad económica en un área se puede dividir en básica -primaria o de exportación, y no-esencial, es decir, secundaria. La relación entre los dos sectores se mantiene constante en el tiempo y los dos sectores están estrechamente vinculados. La teoría postula que los ingresos generados por la demanda externa del sector de base se utilizan para comprar bienes y servicios del sector no-esenciales. Las empresas y los trabajadores del sector noesenciales utilizan esos ingresos para nuevas compras y gastos dentro de la economía local. Este proceso de flujo circular establece un efecto multiplicador en movimiento, de tal forma que impulsar el sector de base desencadena un efecto en toda la economía mucho mayor que el aumento inicial (Andrews, 1953; Blumenfeld, 1955; Richardson, 1969; Williamson, 1975).

A pesar de su atractivo intuitivo, la teoría de la base económica tiene serias limitaciones conceptuales y prácticas. En primer lugar, la división de la economía urbana en sectores básicos y no básicos está plagada de dificultades teóricas y empíricas. En segundo lugar, el énfasis en la importancia de las exportaciones en el fomento de la economía urbana pasa por alto la relevancia del crecimiento endógeno en el desarrollo local y la creciente importancia de los sectores no básicos como son los servicios en una economía avanzada. Por último, y desde una perspectiva de equidad social, al centrarse únicamente en el crecimiento agregado, en términos de ingresos y la evolución del empleo, el modelo no tiene en cuenta el bienestar de la población y los problemas redistributivos.

Adicionalmente, como apunta Graham (2004), el problema con la teoría de la base exportadora como modelo de crecimiento local se encuentra, entre otros aspectos, en que en la actualidad las empresas que exportan fuera del municipio o la región a menudo no reinvierten ni compran sus suministros localmente, no ofrecen necesariamente buenos y permanentes puestos de trabajo, y muchas de ellas suelen des-localizarse en busca de mejores ventajas fiscales o fuerza de trabajo más barato. Aún así, la estrategia de buscar un espacio en la economía global sigue repitiéndose. El principal problema según Graham se encuentra en que desde las teorías económicas, la visión de la base económica es tan aceptada que se hace imposible pensar de forma diferente. Esa incapacidad de pensar diferente crea un grave problema epistemológico, ya que la mayoría de las respuestas económicas que generan académicos, consultores y políticos se enmarcan en esta interpretación estrecha, coercitiva y a la postre, errónea en cuanto a la estrategia de desarrollo local a seguir. 


\section{PENSANDO DESDE LA DIFERENCIA: LA ÓPTICA FEMINISTA Y QUEER}

La crítica feminista ha ejemplificado y discutido ampliamente las limitaciones de la teoría económica tradicional para capturar las dinámicas productivas reales, tal y como ocurre con el trabajo de las mujeres. Así, por ejemplo, ironizando se argumenta que siguiendo la lógica de las categorías económicas aceptadas se puede establecer que si una sirvienta contrae matrimonio con su empleador, ello repercute negativamente en el producto interior bruto de un país ya que lo que se computaba como salario ahora deja de existir.

El esfuerzo de las feministas por hacer visible el trabajo de las mujeres en el hogar y su crítica a indicadores económicos como el Producto Nacional Bruto (PNB) se remonta a las primeras décadas del siglo veinte, con los trabajos de Ilena Bailey (1915), Wesley Mitchell (1921) y Margaret Reid (1934). En su publicación de 1934, The Economics of Household Production, Reid establece el criterio de la "tercera persona". Este criterio que ha sido ampliamente aceptado en estudios que intentan computar el valor productivo de actividades fuera del mercado, establece que es posible identificar un precio por el trabajo realizado dentro del espacio doméstico computando, por ejemplo, cuando costaría si se externalizara fuera del hogar, Así, cocinar, cuidar de los hijos, limpiar la vivienda, etc. tiene un precio de mercado que se puede establecer. Meredith Edwards (1989) amplia el análisis desarrollando un grupo de criterios desde la perspectiva del reemplazamiento, como ya apuntaba Reid, o desde la perspectiva de la oportunidad de coste. Esta oportunidad de coste puede calcularse desde el salario medio de los trabajadores, o, si se quiere ser más preciso y considerando que las mujeres, generalmente, tienen como promedio un salario medio inferior al de los hombres, se podría calcular el coste desde del salario medio de las mujeres en la economía formal; o a partir del salario mínimo interprofesional.

De hecho, la teoría feminista a mediados de los años 1970 empieza a ir va más allá en el esfuerzo por aportar visibilidad y reconocimiento al trabajo de las mujeres. En el contexto de las emergentes teorías posmodernas y posestructuralistas, las feministas reivindican que género no es una categoría esencial del ser humano sino un constructo social. Esto significa que comportarse como un hombre o una mujer no tiene una base biológica que lo hace necesario, como se había argumentado hasta entonces, sino que es algo que se ha diseñado y perpetuado a través de organizaciones sociales.

Al apuntar que las características de género son fruto de las organizaciones sociales, la teoría feminista hace dos contribuciones revolucionarias. Primero separa lo social de lo biológico, insistiendo que si bien lo biológico es algo relativamente estable e inmutable, lo social en cuanto producto de las ideas humanas es mutable. La segunda contribución, a efectos epistemológicos aún más poderosa que la primera, proviene de poder afirmar que en cuanto a constructo social, el género no es esencial a la identidad de un individuo (West y Zimmerman, 1987; Garber, 1992; Lorber, 1994).

La idea humanista de identidad se había caracterizado por considerar que una persona es el producto de algunos aspectos inmutables y esenciales. Así el ser identitario de cada uno respondería, por ejemplo, al tipo de las creencias religiosas o espirituales que 
el individuo tenga -musulmán, judío, católico, agnóstico etc.; al sexo -mujer o hombre; al género -masculino o femenino; a la sexualidad -heterosexual, homosexual, bisexual; a la nacionalidad, etc. La teoría feminista, al apuntar que el género no es esencial al individuo crea una ruptura epistemológica, y junto con las teorías postmodernistas, el ser esencialista e identitario se transforma en subjetividad. En cuanto que sujeto, el individuo puede percibe que su identidad no es innata sino que se crea a través del lenguaje. Ello producirá una nueva ruptura en el feminismo, y por ello, a partir de los años setenta, cuestiones de raza, de etnicidad, de clase social influirán en los estudios feministas. A partir de ahora, con el giro cultural (Soja, 1999), cuando se hable de mujeres ya no se considerará solo a "las mujeres blancas de clase media".

Si la crítica feminista ha establecido que género es un constructo social, la teoría queer, que se inicia a partir de los años noventa desde el análisis de género y sexualidad, cuestiona lo que identifica como el conocimiento dominante, excluyente, dicotómico y binario que domina las ciencias sociales. Apoyándose de nuevo en Foucault, académicos como Butler (1990), Sullivan (2003) y Ahmed (2006) exploran y critican lo que consideran conocimiento normativo rígido; y desde esta posición reabren y amplían el debate sobre la identidad del sujeto. Refutando categorías binarias como hombre/mujer, heterosexual/homosexual, la teoría queer de-construye los modelos teóricos dominantes, los cuales desde esta nueva perspectiva son entendidos como el fruto de un pensamiento y discurso hegemónico hetero-normativo que domina, desde las disciplinas académicas a las narrativas políticas. Esta deconstrucción abre nuevos espacios epistemológicos que permitirán repensar y cuestionar categorías y aproximaciones teóricas hasta ahora bien establecidas que imperan, por ejemplo, en los modelos de desarrollo local y análisis económico.

\section{LA IDENTIFICACIÓN DE NUEVOS ESPACIOS ECONÓMICOS}

A lo largo de su vida académica, Julie Graham publicó más de cincuenta trabajos junto a Katherine Gibson, y lo hicieron de una forma tan creativa como poco convencional en el mundo académico. Crearon un seudónimo/sujeto académico que simboliza su estrecha y creativa colaboración: J.K. Gibson-Graham. Entre sus publicaciones más emblemáticas se encuentran The End of Capitalism (as we knew it) publicado en 1996. En este libro Gibson-Graham utiliza la teoría feminista y la teoría queer para cuestionar el poder hegemónico del discurso económico capitalista.

$\mathrm{Su}$ planeamiento empieza por cuestionarse porqué el discurso del modelo capitalista impera en el análisis de los procesos económicos y en las fórmulas de desarrollo propuestas tanto a macro-escala (países, regiones, etc.) como a escala local. Utilizando el margen de libertad epistemológica que le ofrece la crítica feminista y queer, Gibson-Graham concluye que de hecho, es precisamente el discurso de la tradición marxista el que legitima al capitalismo, presentándolo como un sistema poderoso, dinámico, indestructible y expansivo. De esta forma, el discurso del desarrollo económico queda siempre encapsulado dentro de la dinámica de reproducción del sistema capitalista. Bajo esta perspectiva, el capitalismo puede ser interpretado y entendido -e incluso desde el análisis marxista, puede ser criticado-, 
pero tiene su propia dinámica interna que no puede ser alterada o transformada. Es decir, el discurso dominante sobre el modelo capitalista nos presenta este sistema como fuera de nuestro propio control. Sin embargo, y a pesar de la inhabilidad humana de manejarlo o transformarlo, el modelo capitalista no se interpreta como irracional. Todo lo contrario, la sofisticada narrativa entorno al capitalismo a lo largo de décadas, lo ha legitimado como positivo. La lógica del mercado tiene su propia dinámica interna. El capitalismo se presenta y justifica a través de la posesión de una propia lógica-racionalidad interna.

Siguiendo a Gibson-Graham, la indiscutible racionalidad del sistema capitalista se ha generado asociando la economía a lo masculino. De esta forma se ha ido creado un discurso de metáforas biológicas y fisiológicas que sustentan y legitiman la economía como un proceso de orden racionalidad, en clara oposición a todo lo que no se inscribe dentro del marco del modelo económico capitalista, que se identifica como irracional y desordenado, y que debe por ello ser eliminado o transformado. Además, las metáforas fisiológicas han permitido al capitalismo ser interpretado como un sistema ecológico total que progresa de forma lineal, desde estadios de subdesarrollo o imperfección hacia estadios avanzados y más sofisticados de desarrollo. Un ejemplo de esta interpretación la encontramos en las teorías de Walt Rostow (1960), que explica la teoría del desarrollo a través de la superación de cinco etapas. Aunque esta teoría ha sido ampliamente cuestionada, también ha sido una de las más influyentes en el diseño de estrategias de crecimiento económico, tanto en países desarrollados como en vías de desarrollo.

A través de la posibilidad de cuestionar la visión del sistema capitalista como necesario y "naturalmente" hegemónico, Gibson-Graham por un lado ataca la meganarrativa capitalista y, por otro y más importante, desarrolla la noción de "la economía de la diferencia." Su perspectiva nos permite situamos fuera de la narrativa dominante que presenta la economía capitalista como la única existente. El objetico último para Gibson-Graham es poder imaginar espacios económicos alternativos, para, gracias a ello, poder identificarlos y fomentarlos. Esta apertura a la realidad permite identificar otras prácticas de producción económica importante, como por ejemplo, el trabajo doméstico, el voluntario, o la economía generada desde las cooperativas, las cuales son menoscabadas en el discurso tradicional sobre desarrollo.

$\mathrm{Si}$, The End of Capitalism nos ayuda a interpretar la esfera económica no como un sistema capitalista único, sino como una zona de cohabitación y confrontación de múltiples espacios económicos, es en su libro A Postcapitalits Politics publicado en el año 2006, donde Graham-Gibson avanza en la línea de exponer estos otros espacios económicos múltiples, exponiendo en detalle modos de producción que subsisten junto al modelo capitalista. Para ello identifica nuevos tipos de transacciones y normas existentes fuera del mercado formal que, o bien no son conmensurables, o bien su conmensurabilidad es de carácter social. En el primer grupo se incluyen todas aquellas actividades que no forman parte del mercado, como por ejemplo, trabajos o servicios realizados desde los hogares a través de negociaciones entre sus miembros, ayudas prestadas sin compensación económica resultante de normas culturales de reciprocidad, o los servicios o productos prestados por el sector público a sus 
ciudadanos. Con respecto a la alternativa al mercado que ofrece conmensurabilidad no económica sino social se encuentran, por ejemplo, el comercio justo o el intercambio cooperativo.

También en la esfera laboral Gibson-Graham observa numerosos ámbitos de trabajo cuya compensación no se corresponde con el modelo de salario establecido por la economía de mercado. En primer lugar se explora el amplio abanico de trabajo que se realiza sin ninguna compensación económica, como por ejemplo el trabajo dentro del hogar y el cuidado de la familia, de los que ya habían hablado las feministas de principios de siglo. También existe el trabajo voluntario, el comunitario e incluso el de la esclavitad moderna que se da en numerosos países, tanto en el comercio sexual como en el tráfico de personas, y que se mueve fuera del ámbito de las leyes de mercado capitalista tal y como son interpretadas por la economía clásica. El segundo grupo de actividades laborales no remuneradas, pero muy importantes, son aquellas cuyo salario no se da en términos económicos sino como intercambio alternativo, como por ejemplo el autoempleo, el cooperativismo, la ayuda recíproca, etc.

Finalmente, también existen diferentes formas de negocios cuyo proceso de apropiación de beneficios no opera de la forma capitalista tradicional, repercutiendo en beneficio de los propietarios del negocio o grupos directivos. En primer lugar hay un amplio abanico de empresas de carácter no capitalista cuyos beneficios son de carácter comunal como en el caso de las cooperativas; independiente como en el caso del autónomo; o incluso de esclavitud, en la que no operan las leyes de la oferta y la demanda. Así mismo, hay una esfera amplísima de negocio alternativo al capitalista que incluye todas las actividades del sector público y las organizaciones sin ánimo de lucro, entre otras.

La identificación de amplios mercados, trabajos y empresas que son ignorados por los análisis económicos tradicionales permite cambiar el foco de análisis, dando cabida a la identificación de actividades económicas diversas y espacios económicos que normalmente son ignorados. De esta forma se puede comprender cómo el trabajo asalariado recogido por indicadores como el PNB es solo la punta de un iceberg de lo que es la economía real, la cual es mucho más amplia y diversa.

Uno de los capítulos de A Postcapitalist Politics está dedicado a exponer el caso del modelo de cooperativas de Mondragón. Gibson-Graham califica este tipo de modelo productivo como "economía intencional" en el sentido de que se trata no sólo de actividad económica per se, sino de un proyecto que entiende la economía como un espacio de decisión político y ético. En este punto es importante resaltar la amplia resonancia que la experiencia de Mondragón ha tenido en la literatura anglosajona, comparándola con el interés más limitado que se le ha dando dentro de España.

El caso de Mondragón se analiza enfatizando como este complejo de cooperativas de trabajo con alrededor de 40.000 trabajadores-propietarios es un conglomerado económico de más de 100 fábricas con servicios asociados como banca, hospital, etc. que ofrece un modelo alternativo y viable. Modelo que la ONU eligió como uno de los cincuenta mejores experimentos económico-sociales del mundo. Desde el concepto de política de la posibilidad, ligada a una actitud ética, y a través del papel primordial del ámbito de la comunidad, del modelo Mondragón se destaca el carácter 
subordinado del capital, entendido como elemento subordinado e instrumental para sostener la comunidad. Se presta especial atención a lo que se identifica como "equilibrio", entendido como la necesidad de equilibrar los requisitos de las empresas, con los requisitos de los trabajadores, del medio ambiente, y de los miembros de la comunidad en general. La coordinación de todos estos intereses múltiples implica, en la práctica, un compromiso real a mantener un interminable y creativo proceso de negociación que permite incluir elementos políticos y éticos en la toma de decisiones económicas que escapan así a la "lógica del mercado" que tantas limitaciones ofrece tanto a nivel social como medioambiental.

\section{REFLEXIONES FINALES: UNA SALIDA A LA GLOBALIZACIÓN Y LA CRISIS ECONÓMICA DESDE LA CRÍTICA FEMINISTA}

En las últimas décadas cuando se ha intentado elaborar modelos económicos alternativos se ha acudido a menudo al concepto de desarrollo sostenible como estrategia viable y deseable. Intentar mantener un crecimiento territorial combinando el equilibrio entre medioambiente, economía, y equidad social, a la vez que no comprometer el futuro de generaciones venideras se ha revelado como una idea aglutinadora de voluntades e intereses. Sin embargo, el discurso sobre la sostenibilidad es tan popular como poco efectivo a la hora de solventar los problemas que pretende solucionar (Casellas, 2010). De hecho, como señala Swyngedouw (2007) y Keil (2007), el discurso de la sostenibilidad se ha instrumentalizado dentro del modelo neoliberal, de tal forma que el proceso de acumulación capitalista se ha perpetuado, mientras se ha despolitizado el debate en torno a posibles modelos alternativos de desarrollo. Poli (2010) califica el debate alrededor del concepto de desarrollo sostenible como de fraude. Un fraude resultante del cambio que el movimiento ecologista sufre a raíz de la inclusión del concepto de desarrollo sostenible en el documento Brundtland en 1987 y la posterior conferencia de Rio en 1992. A partir de entonces el problema medioambiental se aborda no desde una cuestión política y ética como se había hecho en un principio, sino como una cuestión técnica, económica y científica, y por tanto dentro de la propia dinámica capitalista. Si hasta principios de los años 1990 el movimiento medioambientalista reclamaba un cambio de modelo productivo y de vida, a partir de entonces el discurso de desarrollo sostenible en cuanto "seudo-ideología" ampliamente aceptada, absorbe y desvirtúa el movimiento verde, neutralizando su posible potencial revolucionario.

Retomando el análisis de género, Buckingham (2007) argumenta que en el presente las cuestiones de desigualdad de género se perpetúan en las estrategias medioambientales existentes, y plantea alternativas centradas en las ideas de microrupturas como estrategias de cambio, las cuales pueden generarse a nivel de microescala, es decir, a escala de barrio o comunidad. Según esta autora, las rupturas protagonizadas por mujeres se materializan a nivel de prácticas y experiencias de pequeña escala que tienen, sin embargo, capacidad de proponer modelos transformadores en relaciones de género y justicia medioambiental. 
En esta línea ahonda el trabajo de Graham en los últimos años. Un trabajo que sintetiza su trayectoria académica centrada en repensar los procesos económicos con el fin de elaborar un lenguaje que permita identificar la diversidad económica existente, enfatizando la gran variedad de transacciones, de formas de trabajo, de tipos de empresas y dinámicas de desarrollo posibles. Todo ello con el fin de elaborar una nueva narrativa y unos nuevos modelos y proyectos de desarrollo que ofrezcan alternativas al modelo capitalista existente. $\mathrm{Y}$ en este objetivo, las economías de pequeña escala, las economías de las comunidades vinculadas a un territorio específico, que utilizan y maximizan las posibilidades que ofrecen los recursos naturales y humanos locales se han identificado como la alternativa viable al proceso de globalización. Una globalización que ha tenido amplias repercusiones negativas en numerosos colectivos, y que además a partir del 2008 ha entrado en una crisis estructural.

Si hasta ahora el proceso de globalización interpretado desde las teorías económicas clásicas nos hablaba de la importancia de los ingresos generados por la demanda externa y de la necesidad de insertarnos en la economía mundial de manera que podamos competir, Graham introduce y elabora una manera de pensar la economía de forma distinta. El nuevo modelo que Graham nos ofrece examina las causas de la desigualdad económica y los sistemas de opresión para revelar que la economía no tiene porque interpretarse como estrategia de crecimiento "per se", sino que debe ser una herramienta útil para que las personas puedan luchar por la justicia social y medioambiental. Si la economía se vincula a la comunidad como un espacio ético y político en que las decisiones de las personas conforman la economía en lugar de seguir las demandas y exigencias del mercado mundial, entonces se puede deslumbrar una posible salida a la crisis existente que no reproduzca las desigualdades sociales y altos costes medioambientales presentes. La idea de economías comunitarias permite pensar la economía de manera diferente y ayuda a visualizar opciones locales gracias a un sentido más inclusivo de lo que implica una economía local. En un periodo de crisis y cambios necesarios, los análisis y propuestas de Julie Graham se convierten en una herramienta conceptual de gran valúa para poder seguir repensando mejores modelos de desarrollo y vías alternativas de futuro.

\section{BIBLIOGRAFÍA}

AHMED, S. (2006): Queer Phenomenology, Orientations, Objects, Others. Durham, Duke University Press.

ARCHER, B.H. (1976): The Anatomy of a Multiplier. Regional Studies 10, 71-77.

ANDREWS, R. B. (1953): Mechanics of the Urban Economic Base: Historical Development of the Base Concept. Land Economics 29, 161-167.

ARNESEN, E. (Ed). (2007): Encyclopedia of U.S. Labor and Working-class History. New York, Routledge. 
BAILEY, I. (1915): A Study of Management of Farm Homes. Journal of Home Economics, 7, 37-38.

BLUMENFELD, H. (1955): The Economic Base of a Community. Journal of the American Institute of Planners 21, 114-132.

BUCKINGHAM, S. (2007): Microgeographies and Microruptures: The Politics of Gender in the Theory and Practice. En KRUEGER, R. y D. GIBBS (eds.): The Sustainable Development Paradox. Urban Political Economy in the United States and Europe, New York: The Guilford Press, 66-94.

BUTLER, J. (1990): Gender Trouble: Feminism and the Subversion of Identity. New York, Routledge.

CASELLAS, A. (2010): La Geografía Crítica y el Discurso de la Sostenibilidad: Perspectivas y acciones. Documents d'Anàlisi Geogràfica, 56 (3), 575-583.

CHIPMAN, J. (1950): The Theory of Intersectoral Money Flows and Income Formulation. Baltimore MD, Johns Hopkins University Press.

EDWARDS M. (1989): Comments on Chapter 2: Household Productive Activities. En Ironmonger, D. (ed.): Households Work: Productive Activities, Women and Income in the Household Economy, Allen \& Unwin, Sydney.

FLORIDA, R. (2002). The Rise of the Creative Class. New York: Basic Books.

FOUCAULT, M. (1980): Power/Knowledge: Selected Interviews and other Writings. New York, Pantheon Books.

FULTON, W. y SHIGLEY, P. (2001): Small Towns Hang On, Planning 67(4), 20-26.

GARBER, M. (1992): Vested Interests: Cross-Dressing and Cultural Anxiety. New York and London, Routledge.

GRAHAM, J. (1988): Post-modernism and Marxism. Antipode 20(1), 60-66.

GRAHAM, J. (2004): Globalization and the Local Economy. Conference at Lancaster Economy Forum: Toward a Research Agenda Saturday, October 30, 2004 http:// www.fandm.edu/x7140

GIBSON-GRAHAM, JK. (1996): The End of Capitalism (As We Knew It): A Feminist Critique of Political Economy. Oxford UK and Cambridge USA, Blackwell

GIBSON-GRAHAM, JK., RESNICK S. y WOLFF, R. (Eds), (2000): Class and Its Others. Minneapolis, University of Minnesota Press.

GIBSON-GRAHAM, JK., RESNICK S. y WOLFF, R. (Eds), (2001): Re/presenting Class: Essays in Postmodern Marxism. Durham NC and London, Duke University Press.

GIBSON-GRAHAM, JK. (2006): A Postcapitalist Politics. Minneapolis, University of Minnesota Press. 
GOODWIN, R. (1949): The Multiplier as Matrix. Economic Journal, 59, 537-555.

GOTTLIEB, P. (1995): Residential Amenities, Firm Location, and Economic Development. Urban Studies 32(9), 1413-1436.

ISARD, W. (1960): Methods of Regional Analysis. Cambridge, MIT Press.

KEIL, R. (2007): Sustaining Modernity, Modernizing Nature: The Environmental Crisis and the Survival of Capitalism. En KRUEGER, R. y D. GIBBS (eds.): The Sustainable Development Paradox. Urban Political Economy in the United States and Europe, New York: The Guilford Press, 41-65.

KIRSCH, M. H. (1998): In the Wake of the Giant: Multinational Restructuring and Uneven Development in a New England Community. Albany, State University of New York Press.

LORBER, J. (1994): Paradoxes of Gender. Connecticut, Yale University Press.

METZLER, L. (1950): A Multiple Region Theory of Income and Trade. Econometrica, $18,329-354$.

MITCHELL, W. (1921): The Making and Using of Index Numbers. Bulletin, 284.

MOSES, N. (2001): Have a Plan, and Make the Most of Arts and Culture. Public Management 83(11), 18-21.

POLI, C. (2010): Sustainable Development: from Fallacy to Fraud. Human Geography: A new radical journal 3 (2) HGvol3no2-WEB05.pdf

REID, M. (1934): The Economics of Household Production. New York, John Wiley and Sons, Inc.

RICHARDSON, H (1969): Regional Economics. New York, Praeger.

ROSTOW, W. (1960): The Stages of Economic Growth: A non-Communist Manifesto. Cambridge, Cambridge University Press.

SOJA, E. W. (1999): Different Spaces: The Cultural Turn in Urban and Regional Political Economy. European Planning Studies, 7. (1), 65-75.

SULLIVAN, N (2003): A critical introduction to queer theory. New York, New York University Press.

SWYNGEDOUW, E. (2007): Impossible Sustainability and the Postpolitical Condition En R. KRUEGER y D. GIBBS (eds.): The Sustainable Development Paradox. Urban Political Economy in the United States and Europe, New York: The Guilford Press, 13-40.

TEAFORD, J. (1994): Cities of the Heartland: The Rise and Fall of the Industrial Midwest. Bloomington, Indiana University Press.

TIEBOUT, C. M. (1956a): Exports and Regional Growth. Journal of Political Economy 64, 160-164. 
TIEBOUT, C. M. (1956b): The Urban Economic Base Reconsidered. Land Economics 31, 95-99.

WEST, C y ZIMMERMAN, D. (1987): Doing Gender. Gender and Society 1, 12551.

WILliAMSON, R. B. (1975): Regional Growth: Predictive Power of the Export Base Theory. Growth and Change 6, 3-10. 